\title{
Microscopic Study of Two Band Superconductivity in Magnesium Diboride Superconductor $\left(\mathrm{MgB}_{2}\right)$
}

\author{
Basanta Kumar Sahoo, ", Biswa Ranjan Mishra ${ }^{2}$, Subhalaxmi Das ${ }^{3}$, Santosh Kumar Barik ${ }^{1}$, \\ Padmaja Patnaik $^{2}$, Ranjan Kumar Bhuyan ${ }^{1}$, Bibekananda Panda ${ }^{4}$ \\ ${ }^{1}$ Post Graduate Department of Physics, Government College (Autonomous) Angul, Angul, India \\ ${ }^{2}$ Department of Physics, Centurion University of Technology and Management, Bhubaneswar, India \\ ${ }^{3}$ Department of Physics, Deogarh College, Deogarh, India \\ ${ }^{4}$ Department of Physics, Saraswati Degree Vidya Mandir Neelakantha Nagar, Berhempur, India
}

Email address:

basanta@iopb.res.in (B. K. Sahoo)

${ }^{*}$ Corresponding author

\section{To cite this article:}

Basanta Kumar Sahoo, Biswa Ranjan Mishra, Subhalaxmi Das, Santosh Kumar Barik, Padmaja Patnaik, Ranjan Kumar Bhuyan, Bibekananda Panda. Microscopic Study of Two Band Superconductivity in Magnesium Diboride Superconductor $\left(\mathrm{MgB}_{2}\right)$. American Journal of Physics and Applications. Vol. 9, No. 2, 2021, pp. 29-33. doi: 10.11648/j.ajpa.20210902.11

Received: March 15, 2021; Accepted: April 7, 2021; Published: April 26, 2021

\begin{abstract}
We formulate a Model Hamiltonian of two band superconductivity for Magnesium Diboride superconductors $\left(\mathrm{MgB}_{2}\right)$. It is a conventional BCS type metallic superconductor which has the highest critical temperature $\mathrm{T}_{\mathrm{c}}=39 \mathrm{~K}$. It is assumed that the superconductivity in $\mathrm{MgB}_{2}$ arises due to metallic nature of the $2 \mathrm{D}$ sheets. From band structure calculations, it is observed that two types of bands i.e. $\sigma$ and $\pi$ bands are located at Fermi surface. Here, we consider phonon mediated superconductivity in which $\sigma$ band is dominant over $\pi$ band i.e. $\sigma$ band is more coupled to a superconductor with much higher coupling. We consider a model Hamiltonian with mean field approach and solve this by calculating equations of motion of Green functions for a single particle. We determine the quasi-particle energy from the poles of the Green functions. We derive the single particle correlation functions and determine the two SC order parameters for both $\sigma$ and $\pi$ band. Here, the two SC order parameters for the bands are solved self- consistently and numerically. The conduction bandwidth $(\mathrm{W})$ is considered as $\mathrm{W}=8 \mathrm{t}_{0}$, where $\mathrm{t}_{0}$ is the hopping integral. To make all the physical quantities dimensionless, we divide $2 \mathrm{t}_{0}$ in each of the physical quantities. We then calculate the gap ratio $2 \Delta(0) / \mathrm{K}_{\mathrm{B}} \mathrm{T}_{\mathrm{c}}$ for both the bands. It is seen form our theoretical model that the two bands of $\mathrm{MgB}_{2}$ superconductors have two different SC gaps with the same critical temperature. We also observe the variation of dispersion curves of quasi-particles for different temperature parameters for both $\sigma$ and $\pi$ band.
\end{abstract}

Keywords: High- $\mathrm{T}_{\mathrm{c}}$ Superconductor, Inter-band Interaction, Mean Field Approximation

\section{Introduction}

One of the common, conventional BCS type of superconductor is Magnesium Diboride $\mathrm{MgB}_{2}$. The superconductivity arises due to Cooper pair mechanism through electron - phonon interaction. The mechanism of these type of superconductors is well explained by BCS type of superconductivity [1]. Its critical temperature of $39 \mathrm{~K}$ is the highest among conventional superconductors and also greater than some of cuprate superconductors where pairing mechanism other than phonons are observed $[2,3]$.
The structure of $\mathrm{MgB}_{2}$ superconductor is hexagonal and the space group is $\mathrm{p} 6 / \mathrm{mmm}$. Here, Boron atoms are arranged like graphite sheets which are segregated by Magnesium atoms. The Boron atoms in $\mathrm{MgB}_{2}$ superconductor form honeycombed layers with magnesium atoms above the centre of hexagons. Specific heat [4, 5] and Tunneling spectroscopy measurements [6], as well as nuclear magnetic resonance (NMR) studies [7] predicts $\mathrm{S}$ - wave type superconductivity in $\mathrm{MgB}_{2}[8,9]$. The presence of isotope effect [11] and pressure dependence of critical temperature [12] predicts the contribution of phono mediated BCS superconductivity. The Fermi surface of $\mathrm{MgB}_{2}$ consists of 
four sheets, two 3D sheets from the $\pi$ bonding with antibonding $\left(\mathrm{B}-2 \mathrm{P}_{\mathrm{z}}\right)$ and other two nearly cylindrical sheets from $2 \mathrm{D} \sigma$ bonding $\left(\mathrm{B}-2 \mathrm{P}_{\mathrm{x}, \mathrm{y}}\right)[13,14]$. Experiments such as point-contact spectroscopy [15], specific heat measurement [4, 5], scanning tunneling microscopy [16] and Raman spectroscopy [17], critical current measurement [18] clearly explain the existence of two distinct superconducting gaps with small gaps $\Delta_{\mathrm{S}}(0)=2.8 \pm 0.05$ $\mathrm{MeV}$ and large gap $\Delta_{\mathrm{L}}(0)=7.1 \pm 0.1 \mathrm{MeV}$ [19]. Both gaps close near the bulk transition temperature $T_{C}=39 \mathrm{~K}$. This case has been predicted theoretically by Liu et al [13]. With $\mathrm{T}_{\mathrm{C}}=39 \mathrm{~K}$ and two distinct superconducting gaps, $\mathrm{MgB}_{2}$ serves as an important test case for Density Functional Theory (DFT) for superconductors.

There is substantial evidence from the band structure calculation that strong covalent bonds are formed between Borons and after the ionization of $\mathrm{Mg}$, two of its electrons is transferred to the conduction band which is formed by Borons [20]. So, it is believed that superconductivity in $\mathrm{MgB}_{2}$ is primarily contributed from 2D metallic sheets of Boron. It is concluded that $\mathrm{MgB}_{2}$ is a typical type II superconductor with Ginsburg-Landau parameter $\mathrm{K} \approx 23$ [21]. It is observed that there is a substantial reduction in isotope effect from the BCS predicted value of 0.5. The critical temperature $\left(\mathrm{T}_{\mathrm{C}}\right)$ in $\mathrm{MgB}_{2}$ depends upon boron substitution whereas there is no appreciable change due to $\mathrm{Mg}$ isotopic substitution. So, the isotopic coefficient due to boron $(\alpha \mathrm{B})$ has a significant role while the contribution due to isotopic coefficient of Magnesium $(\alpha \mathrm{Mg})$ is very small. It is reported by Budko et al about the measured value of $\alpha \mathrm{B}=0.26$ [10]. Hinks et al predicted an $\alpha \mathrm{B}$ of 0.30 and $\alpha \mathrm{Mg}$ of 0.02 [11]. For $\mathrm{MgB}_{2}$, the total isotope coefficient is 0.32 with a high Debye temperature of $\theta_{D}=750 \mathrm{~K}$. Optical measurement [22] and the specific heat measurement [5] roughly estimates the value of $\frac{2 \Delta(0)}{\mathrm{K}_{\mathrm{B}} \mathrm{T}_{\mathrm{C}}} \approx 4.2$ which deviates from the $\mathrm{BCS}$ value of 3.53 [23]. $\mathrm{MgB}_{2}$ is the first material where the multi gap effects are dominant and its transition temperature has been supported with electron-phonon interaction mechanism for the superconductivity. The nature of multiple gaps had been discussed theoretically $[24,25]$. Here, We formulate a twoband Hamiltonian model to study the multi gaps in $\mathrm{MgB}_{2}$ and we use Zubarev type Green function technique [26]. In the introduction, we have reviewed the experimental observations of $\mathrm{MgB}_{2}$ superconductors. We explain the Hamiltonian model where we consider SC pairing mechanism of BCS type in Section - 2. We will define suitable Green functions and find the expressions of SC order parameters for both $\sigma$ and $\pi$ bands. The results of the numerical calculation are discussed in Section-3. Finally, the conclusion is given in Section-4.

\section{Model Hamiltonian and Calculation of SC Order Parameter}

The two band BCS Hamiltonian for our system is

$$
\begin{gathered}
\mathrm{H}=\sum_{\mathrm{k}, \sigma}\left(\epsilon_{\mathrm{k}}^{\mathrm{a}}-\mu\right) \mathrm{a}_{\mathrm{k}, \sigma}^{\dagger} \mathrm{a}_{\mathrm{k}, \sigma}+\sum_{\mathrm{k}, \sigma}\left(\epsilon_{\mathrm{k}}^{\mathrm{b}}-\mu\right) \mathrm{b}_{\mathrm{k}, \sigma}^{\dagger} \mathrm{b}_{\mathrm{k}, \sigma}-\sum_{\mathrm{k}, \mathrm{k}^{\prime}} \mathrm{V}_{\mathrm{aa}}\left(\mathrm{k}, \mathrm{k}^{\prime}\right) \mathrm{a}_{\mathrm{k} \uparrow}^{\dagger} \mathrm{a}_{-\mathrm{k} \mathrm{k}} \mathrm{a}_{-\mathrm{k}^{\prime} \downarrow}^{\dagger} \mathrm{a}_{\mathrm{k}^{\prime} \uparrow}-\sum_{\mathrm{k}, \mathrm{k}^{\prime}} \mathrm{V}_{\mathrm{bb}}\left(\mathrm{k}, \mathrm{k}^{\prime}\right) \mathrm{b}_{\mathrm{k} \uparrow}^{\dagger} \mathrm{b}_{-\mathrm{k} \downarrow} \mathrm{b}_{-\mathrm{k}^{\prime} \downarrow}^{\dagger} \mathrm{b}_{\mathrm{k}^{\prime} \uparrow}- \\
\sum_{\mathrm{k}, \mathrm{k}^{\prime}} \mathrm{V}_{\mathrm{ab}}\left(\mathrm{k}, \mathrm{k}^{\prime}\right) \mathrm{a}_{\mathrm{k} \uparrow}^{\dagger} \mathrm{a}_{-\mathrm{k} \downarrow} \mathrm{b}_{-\mathrm{k}^{\prime} \downarrow}^{\dagger} \mathrm{b}_{\mathrm{k}^{\prime} \uparrow}+\mathrm{cc}
\end{gathered}
$$

Equation (1) represents the total Hamiltonian of our system where both the first and second term represents the Hamiltonian due to hopping of quasi-particles of $\pi$ and $\sigma$ band. Here, $a_{k, \sigma}^{\dagger}\left(a_{k, \sigma}\right)$ represents the creation and annihilation operators for $\sigma$ band and $b_{k, \sigma}^{\dagger}\left(b_{k, \sigma}\right)$ represents the same for $\pi$ band for conduction electrons. One particle kinetic energy for $\sigma$ and $\pi$ band are $\epsilon_{\mathrm{k}}^{\mathrm{a}}$ and $\epsilon_{\mathrm{k}}^{\mathrm{b}}$ with $\mu$ is the chemical potential.

It was assumed that, due to boson exchange, an $\mathrm{S}$ - wave type BCS pairing interaction exists in $\pi$ and $\sigma$ band. The inter band superconductivity of $\sigma$ and $\pi$ band are represented by the third and fourth term of equation (1) respectively. The inter-band pairing interaction for $\sigma$ and $\pi$ are represented by $\mathrm{V}_{\mathrm{aa}}$ and $\mathrm{V}_{\mathrm{bb}}$ respectively. The fifth term represents the Hamiltonian involving inter-band pairing between $\sigma$ and $\pi$ electrons. The inter-band pairing interaction is $\mathrm{V}_{\mathrm{ab}}$.

The Hamiltonian of the equation (1) in the mean-field form is

$$
\begin{gathered}
\mathrm{H}=\epsilon_{\sigma} \sum_{\mathrm{k}}\left(\mathrm{a}_{\mathrm{k} \uparrow}^{\dagger} \mathrm{a}_{\mathrm{k} \uparrow}+\mathrm{a}_{\mathrm{k} \downarrow}^{\dagger} \mathrm{a}_{\mathrm{k} \downarrow}\right)+\epsilon_{\pi} \sum_{\mathrm{k}}\left(\mathrm{b}_{\mathrm{k} \uparrow}^{\dagger} \mathrm{b}_{\mathrm{k} \uparrow}+\mathrm{b}_{\mathrm{k} \downarrow}^{\dagger} \mathrm{b}_{\mathrm{k} \downarrow}\right)-\Delta_{\sigma} \sum_{\mathrm{k}}\left(\mathrm{a}_{\mathrm{k} \uparrow}^{\dagger} \mathrm{a}_{-\mathrm{k} \downarrow}^{\dagger}+\mathrm{a}_{-\mathrm{k} \downarrow} \mathrm{a}_{\mathrm{k} \uparrow}\right)-\Delta_{\pi} \sum_{\mathrm{k}}\left(\mathrm{b}_{\mathrm{k} \uparrow}^{\dagger} \mathrm{b}_{-\mathrm{k} \downarrow}^{\dagger}+\mathrm{b}_{-\mathrm{k} \downarrow} \mathrm{b}_{\mathrm{k} \uparrow}\right)+ \\
\mathrm{V}_{1} \sum_{\mathrm{k}}\left(\mathrm{a}_{\mathrm{k} \uparrow}^{\dagger} \mathrm{a}_{-\mathrm{k} \downarrow}^{\dagger}+\mathrm{a}_{-\mathrm{k} \downarrow} \mathrm{a}_{\mathrm{k} \uparrow}\right)+\mathrm{V}_{2} \sum_{\mathrm{k}}\left(\mathrm{b}_{\mathrm{k} \uparrow}^{\dagger} \mathrm{b}_{-\mathrm{k} \downarrow}^{\dagger}+\mathrm{b}_{-\mathrm{k} \downarrow} \mathrm{b}_{\mathrm{k} \uparrow}\right)
\end{gathered}
$$

Where,

$$
\begin{gathered}
\epsilon_{\sigma}=\epsilon_{\mathrm{k}}^{\mathrm{a}}-\mu, \epsilon_{\pi}=\epsilon_{\mathrm{k}}^{\mathrm{b}}-\mu \\
\Delta_{\sigma}=\mathrm{V}_{\mathrm{aa}}\left\langle\mathrm{a}_{\mathrm{k} \uparrow}^{\dagger} \mathrm{a}_{-\mathrm{k} \downarrow}^{\dagger}\right\rangle=\mathrm{V}_{\mathrm{aa}}\left\langle\mathrm{a}_{-\mathrm{k} \downarrow} \mathrm{a}_{\mathrm{k} \uparrow}\right\rangle \\
\Delta_{\pi}=\mathrm{V}_{\mathrm{bb}}\left\langle\mathrm{b}_{\mathrm{k} \uparrow}^{\dagger} \mathrm{b}_{-\mathrm{k} \downarrow}^{\dagger}\right\rangle=\mathrm{V}_{\mathrm{bb}}\left\langle\mathrm{b}_{-\mathrm{k} \downarrow} \mathrm{b}_{\mathrm{k} \uparrow}\right\rangle \\
\mathrm{V}_{1}=\mathrm{V}_{\mathrm{ab}}\left\langle\mathrm{a}_{\mathrm{k} \uparrow}^{\dagger} \mathrm{a}_{-\mathrm{k} \downarrow}^{\dagger}\right\rangle=\mathrm{V}_{\mathrm{ab}}\left\langle\mathrm{a}_{-\mathrm{k} \downarrow} \mathrm{a}_{\mathrm{k} \uparrow}\right\rangle \\
\mathrm{V}_{2}=\mathrm{V}_{\mathrm{ab}}\left\langle\mathrm{b}_{\mathrm{k} \uparrow}^{\dagger} \mathrm{b}_{-\mathrm{k} \downarrow}^{\dagger}\right\rangle=\mathrm{V}_{\mathrm{ab}}\left\langle\mathrm{b}_{-\mathrm{k} \downarrow} \mathrm{b}_{\mathrm{k} \uparrow}\right\rangle
\end{gathered}
$$

pairing exchange interaction strength $\mathrm{V}_{1} \simeq \mathrm{V}_{2}=\mathrm{V}$. We calculate one electron Green's function given in equation (2) in the super conducting state of $\mathrm{MgB}_{2}$ system. The double time electron Green function is calculated by equation of method [27-29]. The four number of Green's functions $A_{1}(k, \omega), A_{2}(k, \omega), B_{1}(k, \omega)$ and $B_{2}(k, \omega)$ are involved in the calculation.

$$
\begin{aligned}
& \mathrm{A}_{1}(\mathrm{k}, \omega)=\left\langle\left\langle\mathrm{a}_{\mathrm{k} \uparrow} ; \mathrm{a}_{\mathrm{k} \uparrow}^{\dagger}\right\rangle\right\rangle_{\omega} \\
& \mathrm{A}_{2}(\mathrm{k}, \omega)=\left\langle\left\langle\mathrm{a}_{-\mathrm{k} \downarrow}^{\dagger} ; \mathrm{a}_{\mathrm{k} \uparrow}^{\dagger}\right\rangle\right\rangle_{\omega} \\
& \mathrm{B}_{1}(\mathrm{k}, \omega)=\left\langle\left\langle\mathrm{b}_{\mathrm{k} \uparrow} ; \mathrm{b}_{\mathrm{k} \uparrow}^{\dagger}\right\rangle\right\rangle_{\omega}
\end{aligned}
$$




$$
\mathrm{B}_{2}(\mathrm{k}, \omega)=\left\langle\left\langle\mathrm{b}_{-\mathrm{k} \downarrow}^{\dagger} ; \mathrm{b}_{\mathrm{k} \uparrow}^{\dagger}\right\rangle\right\rangle_{\omega}
$$

The equations of motion of the Green function $A_{1}, A_{2}$, $\mathrm{B}_{1}, \mathrm{~B}_{2}$ are

$$
\begin{aligned}
& A_{1}=\frac{1}{2 \pi}\left[\frac{\omega+\epsilon_{1}(\mathrm{k})}{\omega^{2}-\epsilon_{2}(\mathrm{k})^{2}+\left(\Delta_{\sigma}+V\right)^{2}}\right] \\
& A_{2}=\frac{1}{2 \pi}\left[\frac{\omega+\epsilon_{2}(\mathrm{k})}{\omega^{2}-\epsilon_{2}(\mathrm{k})^{2}+\left(\Delta_{\pi}+V\right)^{2}}\right] \\
& \mathrm{B}_{1}=\frac{1}{2 \pi}\left[\frac{-\left(\Delta_{1}+V\right)}{\omega^{2}-\epsilon_{1}(\mathrm{k})^{2}+\left(\Delta_{\sigma}+V\right)^{2}}\right] \\
& B_{2}=\frac{1}{2 \pi}\left[\frac{-\left(\Delta_{2}+V\right)}{\omega^{2}-\epsilon_{2}(\mathrm{k})^{2}+\left(\Delta_{\pi}+V\right)^{2}}\right]
\end{aligned}
$$

The Green functions required for calculating $\mathrm{SC}$ order parameter is $\mathrm{A}_{2}$ and $\mathrm{B}_{2}$. The poles of the Green functions are

$$
\begin{aligned}
& \mathrm{W}_{1,2}= \pm \sqrt{\left(\Delta_{\sigma}+\mathrm{V}\right)^{2}+\epsilon_{1}^{2}(\mathrm{k})} \\
& \mathrm{W}_{3,4}= \pm \sqrt{\left(\Delta_{\pi}+\mathrm{V}\right)^{2}+\epsilon_{2}^{2}(\mathrm{k})} \\
& \Delta_{\sigma}(\mathrm{T})=\mathrm{V}_{0}(\sigma) \mathrm{N}_{\sigma}(0) \int_{-\omega_{\mathrm{D} 1}}^{\omega_{\mathrm{D} 1}} \mathrm{~d}\left[\epsilon_{0}(\mathrm{k})\right] \times\left(\frac{\Delta_{\sigma}+\mathrm{V}}{\omega_{1}-\omega_{2}}\right)\left[\frac{1}{1+\exp \left(\beta \omega_{1}\right)}-\frac{1}{1+\exp \left(\beta \omega_{2}\right)}\right] \\
& \Delta_{\pi}(T)=V_{0}(\pi) N_{\pi}(0) \int_{-\omega_{D 2}}^{\omega_{D 2}} d\left[\epsilon_{0}(k)\right] \times\left(\frac{\Delta_{\pi}+V}{\omega_{1}-\omega_{2}}\right)\left[\frac{1}{1+\exp \left(\beta \omega_{3}\right)}-\frac{1}{1+\exp \left(\beta \omega_{4}\right)}\right]
\end{aligned}
$$

Different physical quantities used in the calculation are superconducting gap parameters $\left(\Delta_{\sigma, \pi}(\mathrm{T})\right)$, the inter pair exchange interaction strength $(\mathrm{V})$, the density of states $\left(\mathrm{N}_{\sigma, \pi}(0)\right)$, the Debye frequencies $\omega_{\mathrm{D}}$ and temperature $(\mathrm{T})$. We divide $2 t_{0}$ in each of the physical quantities involved in the calculation, where $\mathrm{W}=8 \mathrm{t}_{0}$ is the width of the conduction band. The dimensionless parameters are $\mathrm{SC}$ order parameter for $\sigma$ band $\frac{\Delta_{\sigma}(\mathrm{T})}{2 \mathrm{t}_{0}}=\mathrm{Z}_{\sigma}$, SC order parameter for $\pi$ band $\frac{\Delta_{\pi}(\mathrm{T})}{2 \mathrm{t}_{0}}=$ $\mathrm{Z}_{\pi}$, Debye frequency for $\sigma$ band $\frac{\omega_{\mathrm{D} 1}}{2 \mathrm{t}_{0}}=\widetilde{\omega}_{\mathrm{d} 1}$, Debye frequency for $\pi$ band $\frac{\omega_{\mathrm{D} 2}}{2 \mathrm{t}_{0}}=\widetilde{\omega}_{\mathrm{d} 2}$, reduced temperature $\frac{\mathrm{K}_{\mathrm{B}} \mathrm{T}}{2 \mathrm{t}_{0}}=\theta$, Inter pair exchange interaction constant $\frac{\mathrm{V}}{2 \mathrm{t}_{0}}=\mathrm{V}, \mathrm{SC}$ coupling constant for $\sigma$ band $g_{1}=N_{\sigma}(0) V_{0}(\sigma) 3$. and SC coupling constant for $\pi$ band $\mathrm{g}_{2}=\mathrm{N}_{\pi}(0) \mathrm{V}_{0}(\pi)$.

\section{Result and Discussion}

We have self-consistently and numerically solved the SC gaps $\mathrm{Z}_{\sigma}(\mathrm{T})$ and $\mathrm{Z}_{\pi}(\mathrm{T})$. All the physical parameters are in dimensionless form. The dimensionless parameters are SC coupling constant $\left(\mathrm{g}_{1}\right)$ for $\sigma$ band, SC coupling constant $\left(\mathrm{g}_{2}\right)$ for $\pi$ band, SC gap parameter $Z_{\sigma}$ for $\sigma$ band, SC gap parameter $Z_{\pi}$ for $\pi$ band, inter pair exchange interaction constant $(\mathrm{V})$, Debye frequency $\left(\omega_{\mathrm{D}}\right)$ and temperature parameter $(\theta)$. The Fermi level $\epsilon_{\mathrm{F}}$ is in the half position of the conduction band i.e. $\epsilon_{\mathrm{F}}=0$. We consider the conduction bandwidth $\mathrm{W}_{\mathrm{b}}$ where $\mathrm{W}_{\mathrm{b}}=8 \mathrm{t}_{0} \simeq 1 \mathrm{eV}$.

Figure 1 depicts the temperature dependence SC-gap for both $\sigma$ and $\pi$ bands of Magnesium diboride superconductor.
The critical temperature parameter for both the bands is $\left(\theta_{C}\right)=0.1565$ equivalent to $39.1 \mathrm{~K}$. The SC-gap value for $\sigma$ band is $0.0271 \simeq 6.78 \mathrm{MeV}$. The observed gap ratio $\frac{2 \Delta(0)}{\mathrm{K}_{\mathrm{B}} \mathrm{T}_{\mathrm{C}}}=$ $\frac{2 \mathrm{Z}}{\mathrm{K}_{\mathrm{B}} \theta_{\mathrm{C}}}=3.47$ which is slightly less than the universal $\mathrm{BCS}$ value of 3.52. The SC-gap value for $\pi$ band is $0.0145 \sim 3.62 \mathrm{MeV}$ and the gap ratio $\frac{2 \Delta(0)}{\mathrm{K}_{\mathrm{B}} \mathrm{T}_{\mathrm{C}}}=\frac{2 \mathrm{Z}}{\mathrm{K}_{\mathrm{B}} \theta_{\mathrm{C}}}=1.85$. Earlier, Liu et al [30] predicted that the critical temperature for both the bands is $39 \mathrm{~K}$ where our theoretical result gives $39.1 \mathrm{~K}$. The value of $\mathrm{SC}$ gap in $\sigma$ band is in between $6.4 \mathrm{MeV}$ to $7.2 \mathrm{MeV}$ and for $\pi$ band, it is between 1.2 to $3.7 \mathrm{MeV}$ as reported earlier by Choi et al theoretically [31]. The same two SC gaps as reported experimentally range from $5.5 \mathrm{MeV}$ to $8 \mathrm{MeV}$ for $\sigma$ band and $1.5 \mathrm{MeV}$ to $3.5 \mathrm{MeV}$ for $\pi$ band [32-34]. For the observed theoretical result, we have taken the SC coupling constant of $\sigma$ band $\left(g_{1}=0.33\right)$ and the SC coupling constant of $\pi$ band $\left(g_{2}=0.285\right)$. Here we have considered the phonon mediated superconductivity which shows that $\sigma$ band is dominant in $\mathrm{MgB}_{2}$ superconductors with a stronger coupling and $\pi$ band is less coupled to the superconductors with a much weaker coupling which agrees well with the experiment [35 - 37]. We also observed two-bands i.e. $\pi$ band and $\sigma$ band having two different SC-energy band gaps with the same critical temperature of $39.1 \mathrm{~K}$ from our theoretical model calculation and the gap ratio is approximately the same as experimentally and theoretically observed value [30$33,38]$.

Figure 2 depicts the quasi-particle energy plots $\left(\mathrm{W}_{\sigma}\right.$ and $\left.W_{\pi}\right)$ vs. $x_{0}\left(x_{0}=\frac{\epsilon_{0}(K)}{2 t_{0}}\right)$ for temperature parameter $(\theta=0 K)$. Here the quasi particle energy of $\sigma$ band and $\pi$ band are 
$\mathrm{W}_{1}=6.78 \mathrm{MeV}$ and $\mathrm{W}_{3}=3.625 \mathrm{MeV}$ respectively. To study the nature of the plot, we only consider the positive part of quasiparticle energy. These bands show a strong dependence on energy $\epsilon_{0}(K)$. The separation between gaps decreases when the energy $\epsilon_{1}(K)$ increases and shows the dispersion of the $\sigma$ and $\pi$ band which also differ considerably. For $\sigma$ band, the most prominent dispersion was observed and these bands are nearly flat bands. In our model, the plot shows the $\sigma$ band has more prominent dispersion than $\pi$ band. It is clear from figure 2 that the trend of $\sigma$ band shows slightly flat than $\pi$ band at low temperature which agrees well with the experiment $[39,40]$. The dispersed flat band indicates the system as a strongly correlated one. The dispersion of quasi-particles mentioned here agrees well with the band dispersion of quasi-particles reported earlier [41-43].

Figure 3 depicts the variation of the quasiparticle energy band of $\mathrm{W}_{\sigma}$ vs. $\mathrm{x}_{0}$ for different temperature Parameters. At $\theta=0 \mathrm{~K}$, the band shows flat nature. As temperature increases $(\theta=0 \mathrm{~K}$ to $\theta=37.5 \mathrm{~K})$ the dispersion plots shows the same trend but for the temperature $\theta=0 \mathrm{~K}$, the plot is more dispersed than the dispersion plot of other temperature. With the increase in temperature parameter from $\theta=0 \mathrm{~K}$ to $\theta=37.5 \mathrm{~K}$, the quasiparticle energy decreases from $6.78 \mathrm{~K}$ to $1.73 \mathrm{~K}$. However, it is found that the quasiparticle energy depends upon the magnitude of the SC gap at the respective temperature parameters.

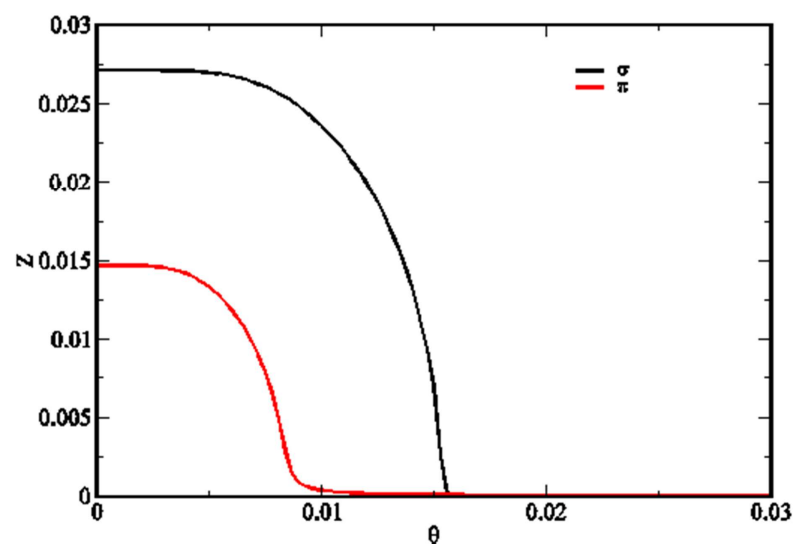

Figure 1. Two SC gaps (z) for $\sigma$ and $\pi$ band $v$ s. temperature parameter $(\theta)$ solved self-consistently. The value of different dimensionless parameters are inter-band pairing constant $(V) \simeq 0.00002$, SC coupling constant of $\sigma$ band $\left(g_{1}=0.33\right)$, SC coupling constant of $\pi$ band $\left(g_{2}=0.285\right)$, Debye frequency for $\sigma$ band $\left(\omega_{d 2}^{\sim}\right)=0.281 \approx 787.7 \mathrm{~K}$, Debye frequency for $\pi$ band $\left(\omega_{d 2}^{\tilde{d} 2}\right)=0.26$ $\simeq 650 \mathrm{~K}$.

Figure 4 depicts the quasiparticle energy of $\pi$ band for the same four temperature parameters. The value decreases from $0.625 \mathrm{MeV}$ to $0.0275 \mathrm{MeV}$ as we move from temperature parameters $\theta=0 \mathrm{~K}$ to $\theta=37.5 \mathrm{~K}$. The energy $\mathrm{W}_{\pi}$ at $\theta=0 \mathrm{~K}$ disperses more than the dispersion curve of the other two temperatures. The separation of dispersion is more prominent at $\mathrm{x}_{0}$ tends to zero and the separation decreases towards higher values of $\theta$.

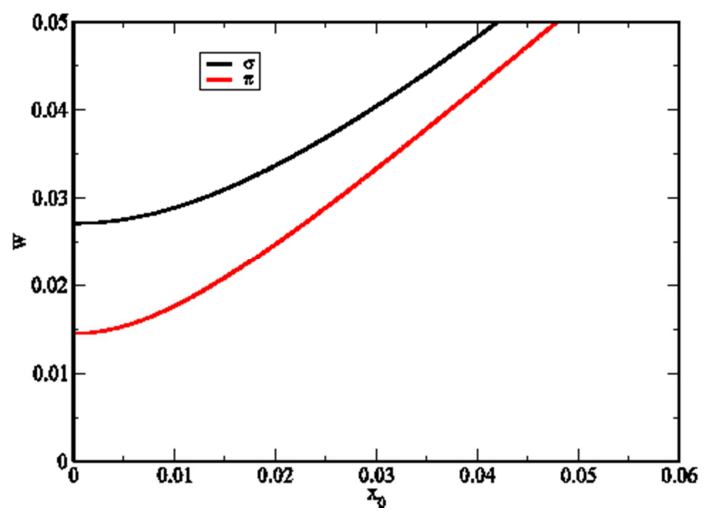

Figure 2. Quasi-particle energy ( $W_{\sigma}$ and $\left.W_{\pi}\right)$ vs. $x_{0}\left(x_{0}=\frac{\epsilon_{0}(K)}{2 t_{0}}\right)$ for temperature parameter $(\theta=0 K)$.

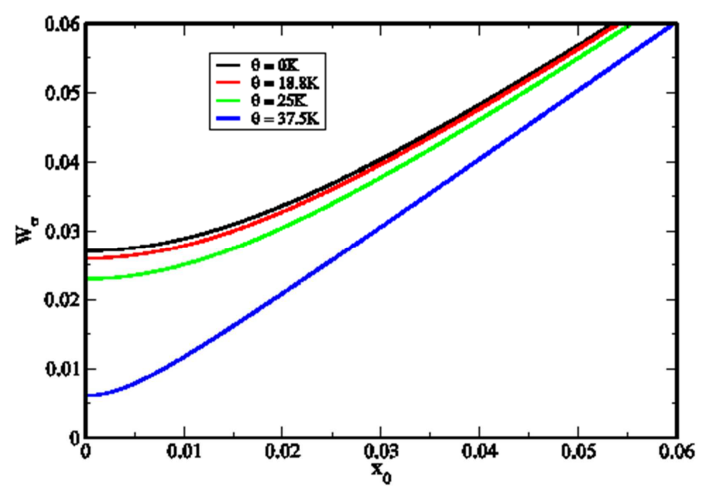

Figure 3. Plot of quasiparticle energy bands of $W_{\sigma}$ vs. $x_{0}$ for different temperature Parameters for $\sigma$ band.

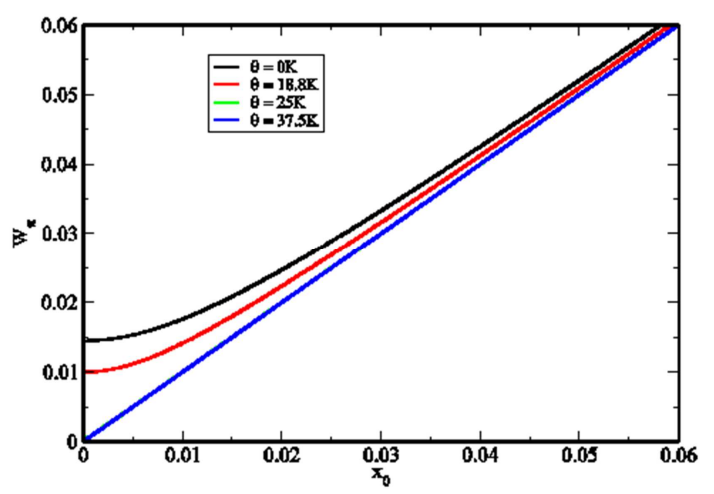

Figure 4. Plot of quasiparticle energy bands of $W_{\pi}$ vs. $x_{0}$ for same four temperature Parameters for $\pi$ band.

\section{Conclusion}

We report two SC-gaps in $\mathrm{MgB}_{2}$ superconductor by taking mean-field levels calculations of Green functions technique. The equation of motions, quasi-particle energy for $\sigma$ and $\pi$ band are derived. We have calculated the correlation functions. The two SC-order parameters for the $\sigma$ band and $\pi$ band are derived. The SC-energy gap and critical temperature for the two bands of $\mathrm{MgB}_{2}$ superconductor are calculated. It is found that the critical temperature for both bands remains the same with two different energy gap which agrees well with the experiment. The nature of dispersion curves of quasi-particle energy is also studied. This model can be improved by adding an external magnetic field in both bands 
to study the SC-gaps in the two-band model.

\section{Acknowledgements}

The authors Basanta Kumar Sahoo, Bibekananda Panda and Subhalaxmi Das are grateful to the Director, Institute of Physics, Bhubaneswar, Odisha, India for providing facilities in the vacation

\section{References}

[1] J. Bardeen, L. N. Cooper, J. R. Schrieffer, Phys. Rev 106162 (1957).

[2] H. B. Schuttler, C. H. Pao, Phys. Rev. Lett. 754504 (1995).

[3] P. W. Anderson, Theory of Superconductivity in the High-Tc Cuprates, Princeton N J (1997).

[4] F. Bouquet, R. A. Fisher, N. E. Philips, Y. Wang, D. G. Hinks, J. D. Jorgenser, A. Junod, Europhys Lett. 56856 (2001).

[5] Y. Wang, T. Plackonwski, A. Junod, Physica C 355179 (2001).

[6] A. Sharoni, I. Felner, O. Millo, Phys. Rev. B 63220508 (2001).

[7] H. Kotegawa, K. Ishida, Y. Kitaoka, T. Muranaka, J. Akimitsu, Phys. Rev. Lett. 87127001 (2001).

[8] S. Hass, K. Maki Phys. Rev. B 65020502 (R) (2001).

[9] Y. Chen, K. Mani Current Applied Physics 1, 4-5, 33 (2001).

[10] S. L. Budko, G. Lapertot, C. Petrovic, C. E. Cunningham, N. Anderson, P. C. Canfield, Phys. Rev. Lett 861877 (2001).

[11] D. G. Hinks, H. Claus, J. D. Jorgensen, Nature 411458 (2001).

[12] G. Karapetrov, M. Iavarone, W. K. Kwok, G. W. Crabtree, D. G. Hinks, Cond-mat/0102312.

[13] A. Y. Liu, I. I. Mazin, J. Kortus, Phys. Rev. Lett 87087005 (2001).

[14] H. J. Choi, M. L. Cohen, S. G. Louie, Physica C 34566 (2003).

[15] H. Schmidt, J. F. Zasadzinski, K. E. Gray, D. G. Hinks, Condmat/0112144 (2002).

[16] F. Giubileo, D. Roditchev, W. Sacks, R. Lamy, D. X. Thanh, T. Kelin, S. Miraglia, D. Fruchart, J. Marcus, Ph. Monod, Phys. Rev. Lett. 87177008 (2001).

[17] X. K. Chen, M. J. Korstantinovic, J. C. Irwin, D. D. Lawrie, J. P. Franck, Cond-mat/0104005.

[18] Glowacki, Bartek A.; Wozniak, Marcin Acta Physica Polontica A; 5 (138), pp. 695-704 (2020).

[19] R. E. Lagos, G. G. Cabrera, Braz. J. Phys. 3314 (2003).

[20] J. Kortus, I. I. Mazin, K. D. Belashchenko, V. P. Antropov, L. L. Boyer, Phys. Rev. Lett. 864656 (2001).
[21] T. S. Kayed, Crystal Research and Technology 39, 50 (2004).

[22] R. da Silva, J. H. S. Torres, Y. Kopelevich, Cond-mat/0105329 (2001).

[23] K. Vinod, N. Verghese, U. Symaprasad, Superconductor Science and technology 20 (10) R31 (2007).

[24] H. Suhl, B. T. Matthis, R. Walker, Phys. Rev. lett, 3, 552 (1959).

[25] J. Kondo, Pro. Theo Phys 8 (1959) 25.

[26] D. N. Zubarev, Sov. Physics. Usp. 95 (1960) 71.

[27] B. K Sahoo, B. N. Panda, Physica C 470, 547 (2010).

[28] B. K Sahoo, B. N. Panda, F. T. Dias, Int. journal of adv. Technology in eng. and science 11 445-450 (2014).

[29] G. C. Rout, S. Das, Physica C 339, 17-26 (2000).

[30] A. Y. Liu, I. I. Mazin, J. Kortus, Cond. mat (2001) 0103570.

[31] H. J. Choi, D. Roundy, H. Sun, M. L. Cohen, S. G. Louie, Nature. 418 (2002) 6899, 758-760.

[32] K. X. Chen, M. J. Konstantinovi, J. C. Irin, D. D. Lowrie J. P. Frank Phys. Rev. Lett. 87, 157002-1-157002-4 (2001).

[33] S. Tsuda, T. Yokoya, T. Kiss, Y. Takano, K. Togano, H. Kito, H. Ihara, S. Shin, Phys. Rev. Lett. 87, 157002-1-157002-4 (2001).

[34] C. Buzea, T. Yamashita Super Cond. Sci. Technol. 14, R115R146 (2001).

[35] H. Schmidt, J. F. Zasadzinski, K. E. Gray, D. G. Hinks, Physics Rev. B 63 (220504) R (2001).

[36] A. Sharoni, I. Felner, O. Millo Physics Rev. B 63 (220508) R (2001) G. Karapetrov, L. Iavarone, W. K. Kwok, G W Crabtree, D. G. Hinks Phys. Rev. lett, 86, 4374 (2001).

[37] G. Karapetrov, L. Iavarone, W. K. Kwok, G W Crabtree, D. G. Hinks Phys. Rev. lett, 86, 4374 (2001).

[38] Hyunsoo Kim, Kyuil Cho, Makariy A. Tanatar, V. Toufour, S. K. Kim, S. L. Budco, V. G. Kogan, R. Projorov, Symmetry, 11 (8) 1012 (2019).

[39] II Mazin and Antropov Physics C 385 (2003) 4.

[40] N. I Medvedea, A L Ivanovskii, J E Medvedeve and A J Freeman Phys Rev. B 64 (2001) 020502.

[41] P. Fulde, Journal of low temperature physics 95 (1994).

[42] B. K Sahoo, B. N. Panda: PRAMAN Journal of Physics 77 (2011).

[43] Ian D. R. Mackinnon, Peter C. TalbotJose, A. Alarco Computational Materials Science 130 (2017) 191-20. 